OPEN ACCESS

Edited by:

Randall J. Cohrs,

University of Colorado, United States

Reviewed by:

Peter Graham Edward Kennedy,

University of Glasgow,

United Kingdom

Nancy M. Sawtell,

Cincinnati Children's Hospital Medical

Center, United States

*Correspondence:

Satish K. Mehta

satish.k.mehta@nasa.gov

Specialty section:

This article was submitted to

Virology,

a section of the journal

Frontiers in Microbiology

Received: 26 October 2018

Accepted: 09 January 2019

Published: 07 February 2019

Citation:

Rooney BV, Crucian BE,

Pierson DL, Laudenslager $M L$ and Mehta SK (2019) Herpes Virus Reactivation in Astronauts During Spaceflight and Its Application on Earth. Front. Microbiol. 10:16. doi: 10.3389/fmicb.2019.00016

\section{Herpes Virus Reactivation in Astronauts During Spaceflight and Its Application on Earth}

\author{
Bridgette V. Rooney ${ }^{1}$, Brian E. Crucian², Duane L. Pierson ${ }^{2}$, Mark L. Laudenslager $^{3}$ and \\ Satish K. Mehta ${ }^{4 *}$
}

${ }^{1}$ GeoControl Systems, Inc., Houston, TX, United States, ${ }^{2}$ NASA Johnson Space Center, Houston, TX, United States, ${ }^{3}$ Anschutz Medical Campus, University of Colorado Denver, Aurora, CO, United States, ${ }^{4}$ Jes Tech, KBR Wyle Laboratories Houston, Houston, TX, United States

Latent herpes virus reactivation has been demonstrated in astronauts during shuttle (10-16 days) and International Space Station ( $\geq 180$ days) flights. Following reactivation, viruses are shed in the body fluids of astronauts. Typically, shedding of viral DNA is asymptomatic in astronauts regardless of mission duration; however, in some cases, live/infectious virus was recovered by tissue culture that was associated with atopic-dermatitis or skin lesions during and after spaceflight. Hypothalamicpituitary-adrenal (HPA) and sympathetic-adrenal-medullary (SAM) axes activation during spaceflight occurs as indicated by increased levels of stress hormones including cortisol, dehydroepiandrosterone, epinephrine, and norepinephrine. These changes, along with a decreased cell mediated immunity, contribute to the reactivation of latent herpes viruses in astronauts. Currently, 47/89 (53\%) astronauts from shuttle-flights and 14/23 (61\%) astronauts from ISS missions shed one or more herpes viruses in saliva/urine samples. Astronauts shed Epstein-Barr virus (EBV), varicella-zoster virus (VZV), and herpes-simplex-1 (HSV-1) in saliva and cytomegalovirus (CMV) in urine. Larger quantities and increased frequencies for these viruses were found during spaceflight as compared to before or after flight samples and their matched healthy controls. The shedding did not abate during the longer ISS missions, but rather increased in frequency and amplitude. These findings coincided with the immune system dysregulation observed in astronauts from shuttle and ISS missions. VZV shedding increased from $41 \%$ in space shuttle to 65\% in ISS missions, EBV increased 82 to $96 \%$, and CMV increased 47 to $61 \%$. In addition, VZV/CMV shed $\leq 30$ days after ISS in contrast to shuttle where VZV/CMV shed up to 5 and 3 days after flight respectively. Continued shedding of infectious-virus post-flight may pose a potential risk for crew who may encounter newborn infants, seronegative adults or any immunocompromised individuals on Earth. Therefore, developing spaceflight countermeasures to prevent viral reactivation is essential. Our spaceflightdeveloped technologies for saliva collection/rapid viral detection have been extended to include clinical applications including zoster patients, chicken pox, post-herpetic neuralgia, multiple sclerosis, and various neurological disorders. These protocols are employed in various clinics and hospitals including the CDC and Columbia University in New York, as well as overseas in Switzerland and Israel.

\footnotetext{
Keywords: herpes, latency, viral reactivation, spaceflight, immunity
} 


\section{INTRODUCTION}

\section{Herpes Virus}

Herpes viruses have co-evolved with humans for millennia and subsequently employ sophisticated strategies to evade the host immune response. Consequently, after primary infection, they persist lifelong in a latent or dormant phase, and are generally asymptomatic in immunocompetent individuals. However, they may reactivate during periods of increased stress, isolation, and during times of immune challenge. Eight major herpes viruses parasitize humans with worldwide infection rates of $70-95 \%$. Four of the eight are shed in the body fluids of NASA astronauts during both short and long duration spaceflight. Though viral load (virus detected in the body fluids) can be high, these astronauts often have no clinical symptoms associated with reactivation (Mehta et al., 2004). Post-reactivation, replication of the virus may also be enhanced which could account for the significant increase in viral shedding during spaceflight. Yet, there have been a few cases where the reactivation culminated in commensurate atopic dermatitis and/or viral lesions (Crucian B. E. et al., 2016).

\section{Astronaut Stress/Exposures}

Exposure of astronauts, during both short and long duration spaceflight, to non-terrestrial hazards such as variable gravitational forces including acceleration/deceleration, cosmic radiation, and microgravity result in a unique set of stressors that contribute to the dysregulation of the immune and endocrine systems (Crucian and Sams, 2009; Crucian et al., 2013). In addition, they also endure some common stressors including but not limited to social separation, confinement, sleep deprivation, circadian rhythm disruption, and anxiety. There is increasing evidence to suggest that these spaceflight-associated stressors chronically amplify the release of stress hormones, which negatively affects the immune system, especially the adaptive immune system facilitating latent herpes virus reactivation during and after spaceflight. Increased levels of salivary, plasma and urinary stress hormones such as cortisol and catecholamines commonly accompany spaceflight (Stowe et al., 2001a).

\section{Altered Immunity}

Maintenance of viral latency requires a vigorous and vigilant immune system, highly dependent upon competent cytotoxic T-cells, and any changes in immune status tend to promote viral reactivation. This is evident in both terrestrial space-analog studies (Crucian B. et al., 2014) and spaceflight studies (Stowe et al., 2001b, 2011; Mehta et al., 2014, 2017). The alterations in immune status for terrestrial analog studies are minor and coincide with mild viral reactivation. Spaceflight studies illustrate major immune dysregulation and functional changes in conjunction with significant viral reactivation, regardless of mission duration. In fact, substantial changes in cell-mediated immunity exist in most astronauts that reactivated one or more herpes viruses (Mehta et al., 2014; Crucian et al., 2015). This was also highlighted by Glaser et al. (1993), who previously showed an association of EBV reactivation and diminished cell-mediated immunity.
The hypothalamus-pituitary-adrenal (HPA) axis along with the sympathetic-adrenal-medullary (SAM) axis partially mediate the stress response where glucocorticoids and catecholamines are secreted in proportionate concentrations relative to the stress stimulus (Figure 1) (Padgett and Glaser, 2003; Webster and Glaser, 2008; Goldstein, 2010). Though acute responses to stress can be positive, long duration or chronically high levels of stress hormones can negatively affect the regulation of the immune system and its individual components (Crucian B. E. et al., 2014). Changes in a variety of immune cells, both in form (phenotype) and function (killing capacity), result in decreased cell-mediated immunity, which facilitates opportunistic reactivation of latent viruses (Crucian et al., 2015; Bigley et al., 2017).

\section{Stress Hormones/Cytokines}

Cortisol and dehydroepiandrosterone (DHEA) are glucocorticoid steroid hormones released by the adrenal glands in response to stress. Cortisol is anti-inflammatory and immunosuppressive, but DHEA is an important antagonist to cortisol. For that reason, the molar ratio of cortisol to DHEA $[\mathrm{C}] /[\mathrm{D}]$ is an important indicator of immune regulation. In recent flight studies, the regular diurnal release of these hormones was tracked in saliva samples to evaluate any changes/trends occurring through the various phases of flight; launch/pre-flight, flight, and return (Mehta et al., 2014). Salivary cortisol was present in significantly higher concentrations in samples taken before and during flight. Salivary DHEA followed its normal daily decline kinetics in the samples taken before, during and after flight, but has been found to have significantly lower waking concentrations during the flight phase in comparison to samples taken before and after flight. Altogether, diurnal patterns of salivary cortisol were significantly higher during flight while DHEA was significantly lower. The cortisol area under the curve relative to ground (AUCg) did not change significantly during flight relative to baseline whereas DHEA AUCg significantly declined during flight relative to baseline. Ultimately, this results in an increased [C]/[D] molar ratio during spaceflight (Figure 2) which potentially indicates immune challenge, and has been linked to immune modulation (Christeff et al., 1997), including the increased inflammatory cytokine response and the TH2 shift observed in earlier spaceflight studies (Mehta et al., 2013a; Crucian B. E. et al., 2014).

Cytokines are small cell-signaling proteins that play a crucial role in the modulation of the human immune response. They can facilitate both pro- and anti-inflammatory immune states and are generally analyzed in the categories of inflammatory cytokines (IL-1 $\alpha$, IL-1 $\beta, \mathrm{TNF} \alpha$, IL-6, IL-8), lymphoid growth factors (IL-2, IL-7, IL-15), Th1/17 cytokines (IFN $\gamma$, IL-12, IL-17), Th2 cytokines (IL-4, IL-5, IL-10, IL-13), myeloid growth factors (G-CSF, GM-CSF), and chemokines (eotaxin, MCP-1, M1P1 $\alpha$, IP-10). Recent flight studies (Mehta et al., 2013a; Crucian B. E. et al., 2014; Crucian et al., 2015) have shown that astronauts displayed significant increases in the pro-inflammatory plasma cytokines IL-1 $\alpha$, IL-6, IL-8, IFN $\gamma$, IL-4, eotaxin, and IP-10 in samples taken 10 days before launch (L-10), in comparison to their baseline samples taken 180 days before launch (L-180). The increase of IL-6, IL-8, IL-4, eotaxin, and IP-10 is also evident 


\section{Stress Immune Response}

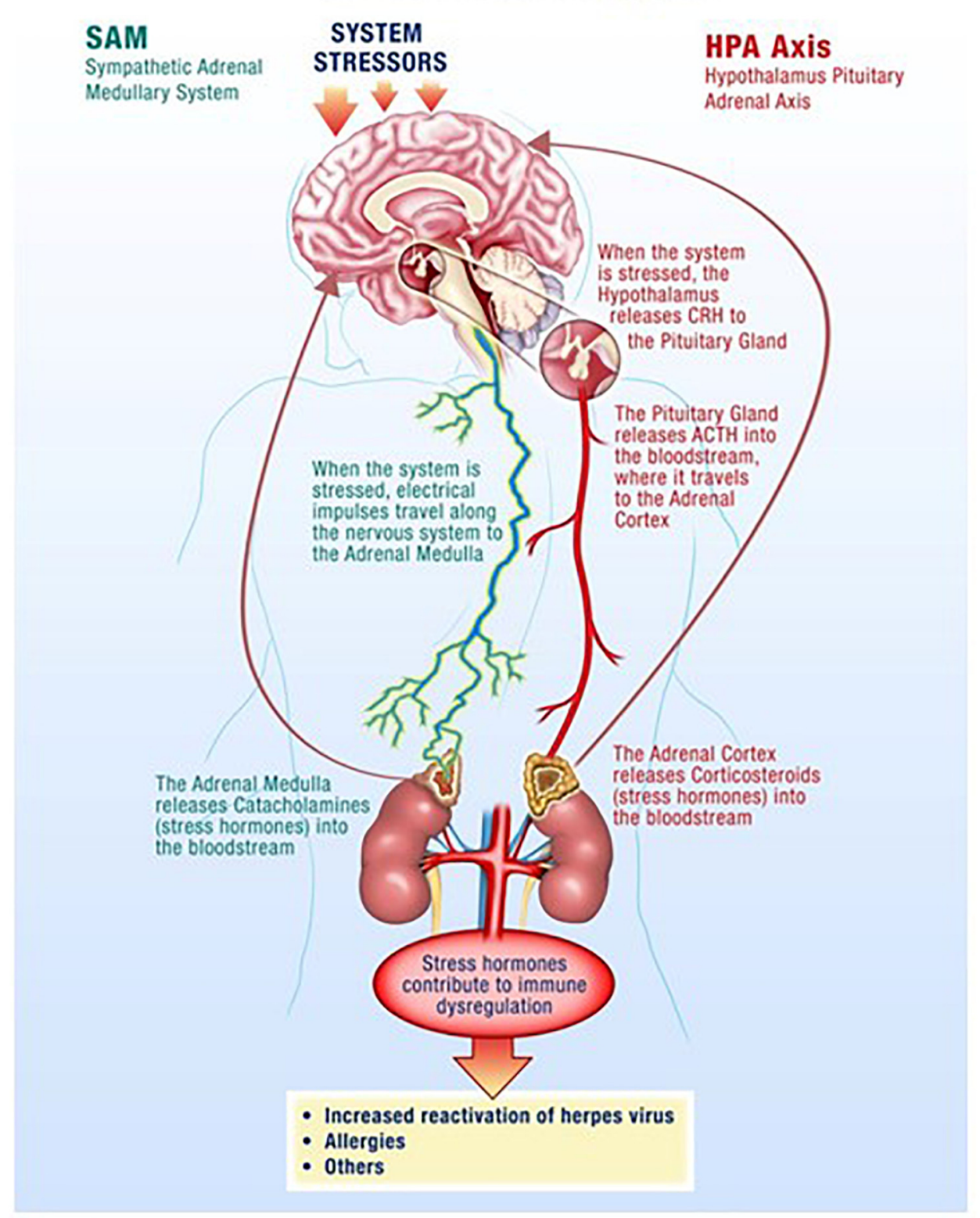

FIGURE 1 | Spaceflight is a stressful environment with various stressors acting through the hypothalamus-pituitary-adrenal (HPA)-axis and the sympathetic-adrenal-medullary (SAM)-axis. Increases in stress hormones, such as cortisol from the adrenal glands, result in reductions in cellular immunity which facilitates opportunistic viral reactivation.

immediately upon return to Earth at landing, designated as $\mathrm{R}+0$. The Th2 cytokine IL- 4 was the most sensitive/responsive to the phases of flight with 35- and 21-fold increases from baseline values at $\mathrm{L}-10$ and $\mathrm{R}+0$, respectively.

When analyzing plasma cytokine levels in the context of virus shedding, there seems to be a connection between astronauts who shed virus and significantly elevated levels of cytokines (IL$1 \alpha$, IL-6, IL-8, IFN $\gamma$, IL-12p70, IL-4, IL-10, IL-13, eotaxin, and IP-10) (Mehta et al., 2013a). Lymphoid and myeloid growth factors are also elevated in virus shedding astronauts, by about twofold. As mentioned earlier, the Th2 cytokine IL- 4 shows the largest fold increases through launch and return flight phases, and this is evident again when restricting the analysis to only viral-shedding astronauts at the return time point $\mathrm{R}+0$. For these astronauts, the single largest plasma cytokine increases were IL-4 (21-fold increase) and IL- 6 (33-fold increase). This indicates a dynamic shift from a Th1 antiviral immune state to a Th2 antibacterial/antifungal immune state. Further emphasizing the Th1-Th2 shift is an analysis of the ratio of IFN $\gamma$ : IL-4. The results from some of the most recent flight studies suggest 


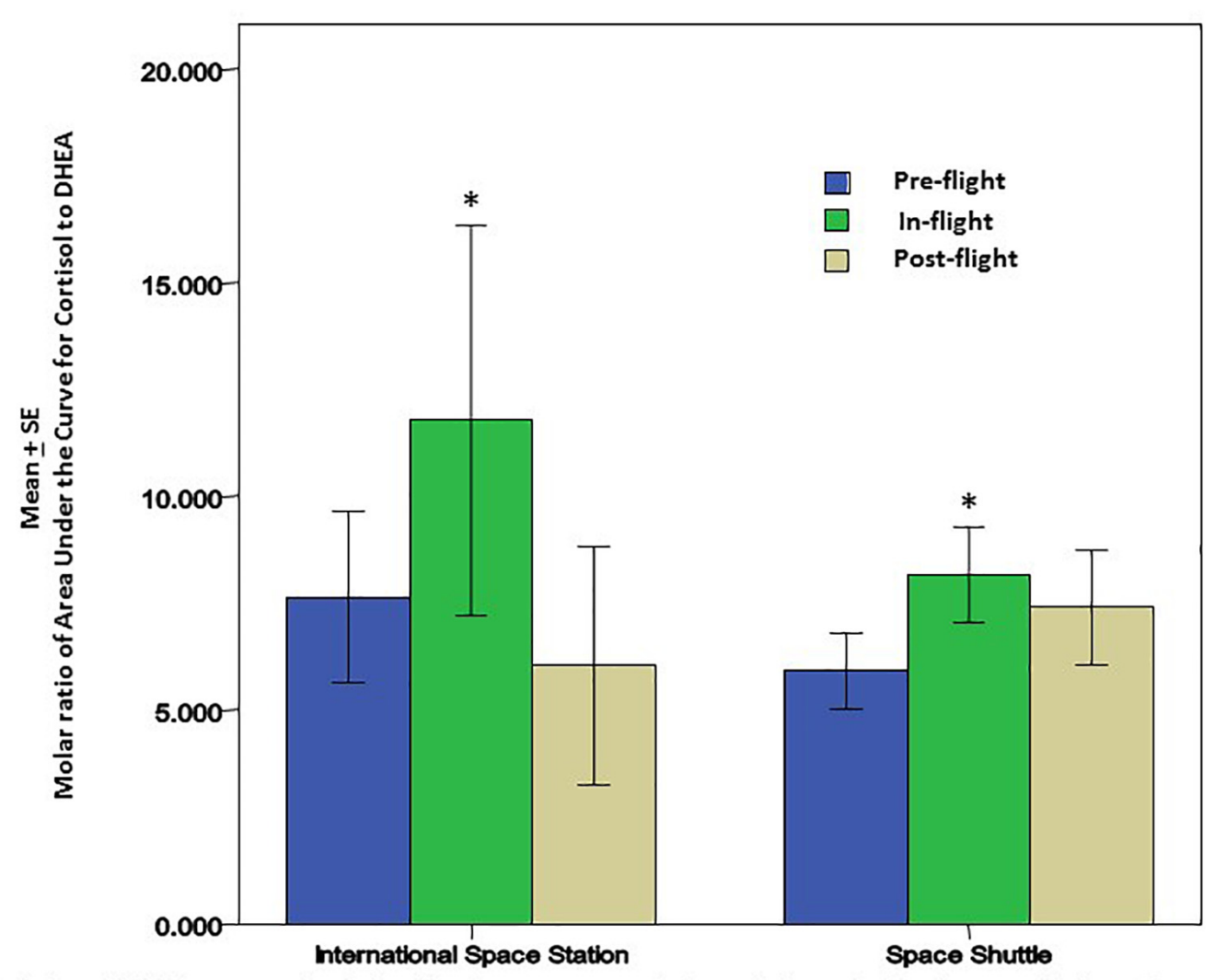

FIGURE 2 | Cortisol and DHEA were analyzed in saliva from astronauts before, during and after the space flights using a commercially available ELISA assays (Salimetrics, LLC, State College, PA, United States). There was a significant increase in the molar ratio of cortisol to DHEA during the flight phase for both Space Shuttle $(N=17)$ or ISS $(N=10)$. The increase in this ratio may be associated with lower cellular immunity and innate immunity; potentially contributing to greater inflammatory cytokines that may affect bone remodeling and bone growth. ${ }^{*}$ Indicates significance when comparing flight against pre-flight and post-flight. $p<0.01$.

a significant decrease in the IFN $\gamma$ : IL-4 ratio for shedders compared to astronauts who did not shed any viruses during their duty rotation (Mehta et al., 2013a; Crucian B. E. et al., 2014).

\section{Viral Specific T-Cell and NK-Cell Function}

Alterations in the aforementioned cytokines play a critical role in the fate of many important leukocyte populations. The cytokine profile changes, acting either independently or in conjunction with microgravity, generate a variety of immune vulnerabilities by significantly changing the numbers, proportions, and functions of leukocytes. Monocyte (Kaur et al., 2005), granulocyte (Stowe et al., 1999), and lymphocyte functions (Crucian et al., 2015; Bigley et al., 2017) are diminished, critically reducing the effectiveness of the immune response to pathogens, as well as its capacity to prevent viral reactivation. T-Cells and NK-Cells in particular, which function to attack and destroy viruses/virally infected cells, are substantially debilitated during spaceflight. Flight studies focusing on T-Cell function have elucidated that both $\mathrm{CD} 4+$ and CD8+ T-Cells taken from astronauts during the flight phase respond ineffectively against a variety of stimuli. Under normal circumstances, these same stimuli would have elicited a more profound response by the T-Cells. The weakened response can last the duration of the flight phase (Crucian et al., 2015). Additional flight studies focusing on the function of NK-Cells have shown decrements in cytotoxicity due to decreased production of the enzymes perforin and granzyme B (Bigley et al., 2017). Without these enzymes, NK-Cells are rendered ineffective against the target cell/pathogen and this impairment may last up to 60 days post-flight. In both cases, reductions in T-Cell and NK-Cell function lead to the inability of the immune system to suppress/sequester/eliminate opportunistic viral reactivation.

\section{Viral Latency}

As stated earlier, herpes viruses share a long-term co-evolutionary history with humankind. This promotes a relatively benign lifelong persistence of the virus within the host. In healthy individuals with robust immune surveillance, viral activity can occur in the absence of clinical symptoms (Grinde, 2013). Injury to the host is antithetical to viral survival. Viral persistence in the host is aided by viral strategies for latency. Latency is a well-orchestrated series of concomitant events that allow for viral genome maintenance, while actively repressing lytic (replicative) gene expression and promoting latent 
gene expression. A hallmark of viral latency is that infectious viral progeny are not produced, so the surrounding cells remain uninfected or naive. Viral latency is the culmination of a handful of factors; infection of cell types permissive to latency, viral promotion of infected cell survival, and the general evasion of the host immune response. Cellular tropism is dictated by cell surface receptor expression, as well as intracellular conditions permissive to viral activity, and is very specific to the individual herpes viruses. HSV and VZV infect neurons in ganglia, while EBV and CMV preferentially infect the cells of the immune system, B cells and myeloid progenitor cells, respectively. Other cell types can be infected by the viruses, but the aforementioned cell types serve as the greater viral latent reservoirs. The promotion of infected cell survival is the product of viral manipulation of host cell machinery. For example, the manipulation of $\mathrm{Bcl}-2$ family proteins promotes survival of $\mathrm{CMV}$ infected monocytes (Collins-McMillen et al., 2015). Not only can herpes viruses manipulate the cells they infect, but they can also affect the host immune response. Interestingly, latent viruses are still very genetically active even in the absence of replication. There is emerging evidence of significant miRNA activity during latency that can act to override lytic transcription, as well as to alter the cell secretome. Though the role of miRNA are yet to be fully teased out, they seem to facilitate the transcription of proteins that mimic host cytokines and chemokines which ultimately inhibit host anti-viral activity. For the many nuances of viral latency, specific to each virus, the reader is directed to the following review articles (Eshleman et al., 2011; Nicoll et al., 2012; Kempkes and Robertson, 2015; Wills et al., 2015).

\section{Viral Reactivation}

Reactivation and shedding of latent herpes viruses has been reported in astronauts during space shuttle, Russian Soyuz and International Space Station missions (Pierson et al., 2005, 2007; Mehta and Pierson, 2007; Mehta et al., 2014, 2017). Virus reactivation has also been observed in ground-based models of spaceflight including Antarctica, undersea habitat, artificial gravity and bed rest studies, though not to the extent seen during spaceflight studies. So far, 47 out of 89 (53\%) astronauts from short duration space shuttle flights, and 14 out of 23 (61\%) from long duration ISS spaceflight missions shed at least one or more herpes viruses in their saliva or urine samples. Significant reactivations of EBV, CMV, and VZV occurred during flight phase and the magnitude and frequency of viral shedding during spaceflight directly correlates with duration of spaceflight. VZV shedding increased from $41 \%$ in space shuttle to $65 \%$ in ISS missions, EBV increased from 82 to $96 \%$, and CMV increased from 47 to $61 \%$. In addition, VZV and CMV shed up to a month post-long duration flight. Percent distribution of these viruses during shuttle and ISS missions is depicted in Figure 3. These viruses often reactivate in concert with one another, but they may also reactivate independently of the other viruses. Reactivation of latent viruses during long-duration spaceflight could increase risk for adverse medical events during exploration-class deepspace missions (Crucian and Sams, 2009). Taken altogether, and to our knowledge, there have been six incidences of astronauts with complaints of symptoms related to herpes viral reactivation (Crucian B. et al., 2016). VZV is an important health risk to crewmembers (several have experienced shingles during flight). Furthermore, CMV can be immuno-suppressive and may play

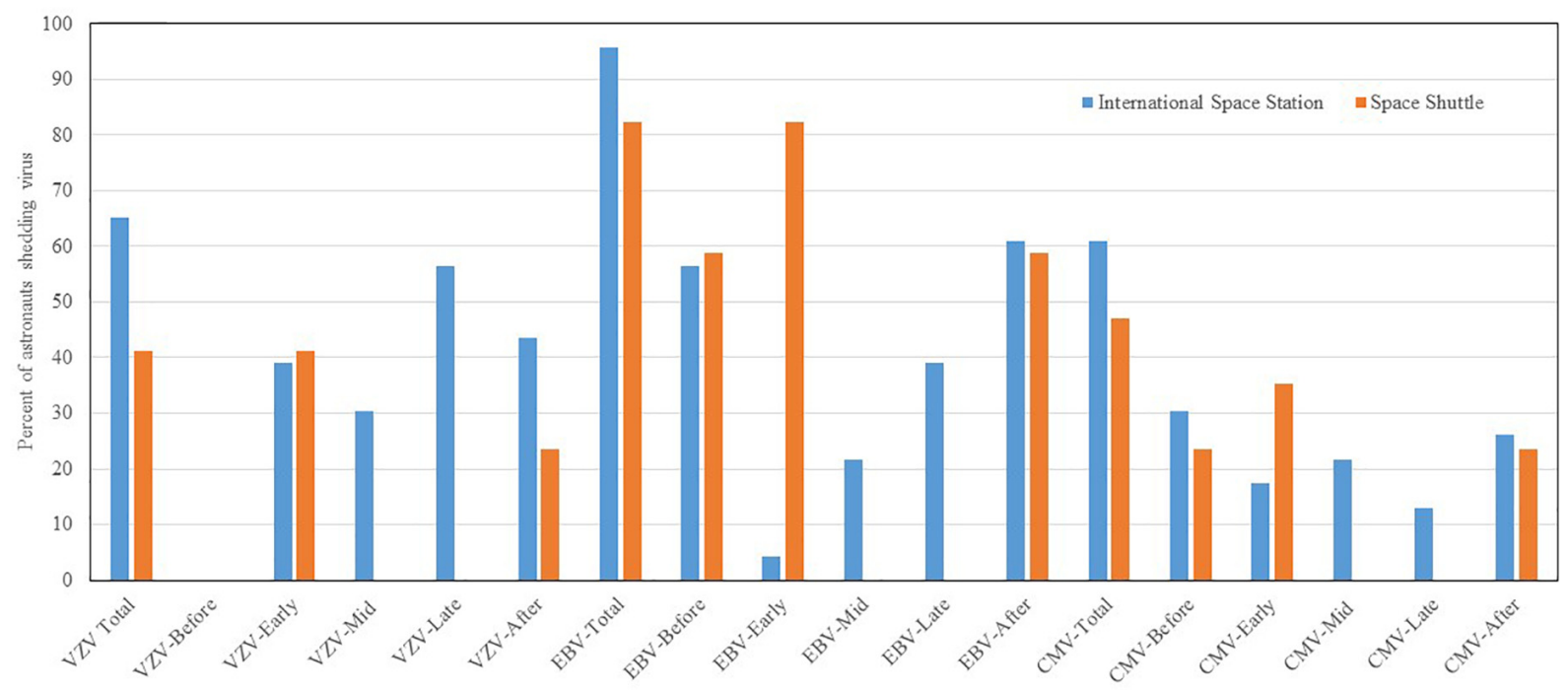

FIGURE 3 | Percent distribution of astronauts shedding VZV. EBV and CMV before, during the mission at time point early-mission, mid-mission, late-mission, and after either short and long duration space flights. Saliva and urine samples were collected from 112 astronauts (89 short duration and 23 long duration) before, during, and after the spaceflight. Saliva was analyzed for Epstein-Barr virus (EBV) and varicella-zoster virus (VZV), and urine was analyzed for Cytomegalovirus CMV by real time PCR assay using Taqman 7900 (Thermofisher, Inc.). The shedding of EBV. VZV and CMV DNA in body fluids is significantly higher during spaceflight as compared to pre-flight, post-flight, and the control $p<0.01$ (Mehta et al., 2014, 2017). However, when comparing these shedding patterns between space shuttle and ISS missions, the differences were not significant. 
a role in the well-documented immune dysfunction observed in crewmembers.

\section{Epstein-Barr Virus}

Epstein-Barr virus (EBV) is responsible for infectious mononucleosis and is associated with several malignancies (Niedobitek et al., 2001; Thompson and Kurzrock, 2004; Bravender, 2010). It is a highly infectious DNA virus transmitted by aerosolized micro-droplets and by direct contact with saliva. It has a 95\% infection rate among adults worldwide, which makes it an ideal target for investigation among a limited and unique astronaut population. EBV preferentially infects B-lymphocytes and these cells serve as a latent virus reservoir. Early flight studies from the shuttle missions were the first to demonstrate that EBV DNA was shed in astronaut saliva samples taken before, during, and after space flight (Stowe et al., 2001b; Pierson et al., 2005; Mehta et al., 2014). These studies highlighted a 10-fold increase in viral load during the flight phase in comparison to samples taken before or after flight. Additionally, EBV copies shed during space flight seemed to increase as a function of time in space, and as a result of diminished cell immunity (Mehta et al., 2000a). These early findings have been repeatedly corroborated in longer duration ISS missions (Stowe et al., 2011; Mehta et al., 2017). Altogether, flight studies have illustrated that approximately $90 \%$ of astronauts, regardless of mission duration, shed EBV during spaceflight.

\section{Varicella Zoster Virus}

Varicella Zoster virus (VZV) is highly communicable and responsible for both chicken pox (primary infection) and shingles (secondary infection). The virus is transmitted via saliva and can be aerosolized by sneezing and coughing. After primary infection,

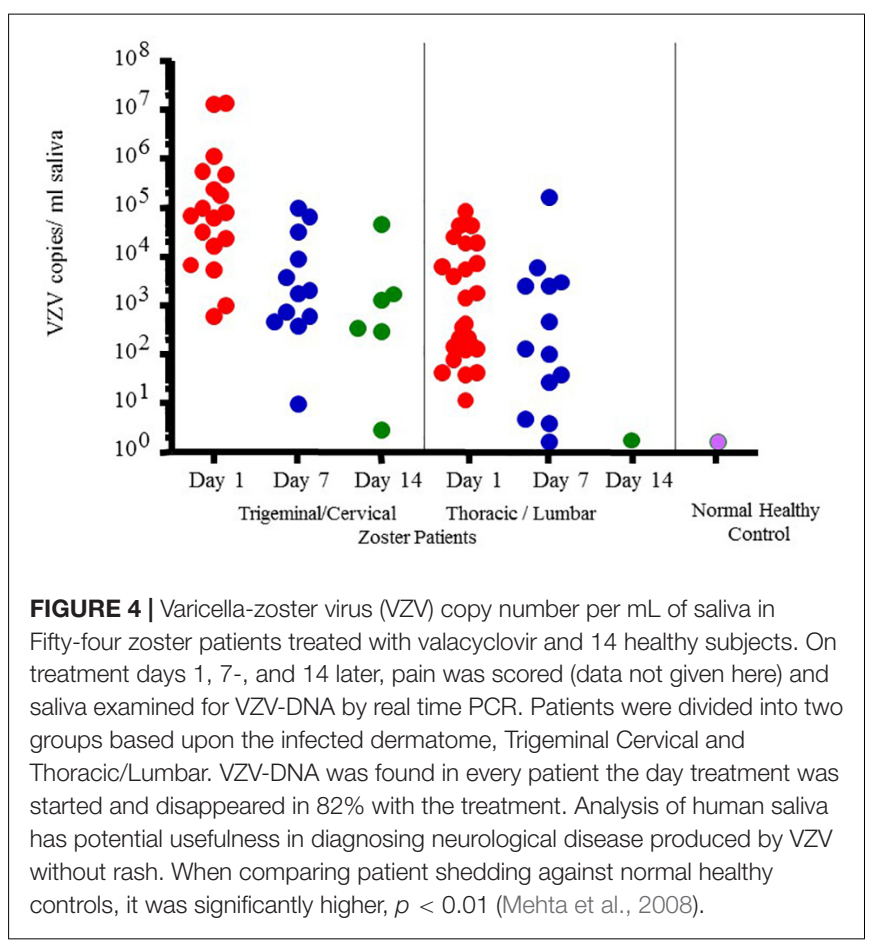

VZV becomes latent in various nerve ganglia (Reichelt et al., 2008) (cranial, dorsal root, autonomic) along the entire length of the neuroaxis, and reactivation often results in characteristic skin lesions that range from aggravating to painful. Though reactivation of VZV is evident from flight studies where viral DNA was found in saliva of astronauts from both shuttle and ISS missions, astronauts do not often develop symptoms or rash (Mehta et al., 2004; Cohrs et al., 2008). Additionally, we have found that saliva samples taken 2-6 days following landing were infectious by culturing that saliva with human fetal lung (HFL) cells. Infectious VZV was present and confirmed by visual inspection of the culture where viral plaques were obvious, as well as by antibody staining and real-time PCR DNA analysis. This poses a risk to the welfare of both astronauts and their seronegative contacts back on Earth, as VZV viral load also increases with time in space and is present in saliva of about $60 \%$ of astronauts from combined shuttle and ISS missions.

\section{Herpes Simplex Virus-1}

Herpes Simplex virus-1 is highly prevalent and communicable and persists as a latent virus lifelong. Generally referred to as oral herpes, reactivation can be either asymptomatic or lead to lesions/rash anywhere on the body. Incidences of HSV1 reactivation are very low with only $8 \%$ of astronaut saliva samples test positive for viral DNA, though recently, an astronaut suffering persistent dermatitis during a long duration spaceflight, $>180$ day, was positive for HSV-1 viral DNA in saliva and lesion samples. The saliva containing virus was infectious, as evidenced by a culture of the saliva atop HFL cells, where visual disruption of HFL cells was apparent at 3 days post-infection with saliva, with viral load verified/quantified by real-time PCR.

\section{Cytomegalovirus}

Cytomegalovirus is the only beta-herpes virus known to reactivate in astronauts. It is typically acquired asymptomatically during childhood and has a worldwide prevalence of 75$90 \%$. Though it remains asymptomatic in immunocompetent people, it may reactivate in individuals whose immune systems are either immature or immunocompromised causing multiple diseases such as encephalitis, gastroenteritis, pneumonia, and chorioretinitis (Seitz, 2010). Moreover, several studies have suggested that CMV infection is immunosuppressive because it directly infects leukocytes as well as hematopoietic cells (Varani and Landini, 2011; O'Connor and Murphy, 2012; Stevenson et al., 2014). Additionally, CMV has been uniquely linked to early immune senescence (Pawelec, 2014; Sansoni et al., 2014). However, a study was able to illustrate a benefit of CMV infection specifically to young adults (2030 years old) regarding increased antibody response to the influenza vaccine (Furman et al., 2015). Spaceflight studies have shown that $27 \%$ of the astronauts from short-term space missions shed CMV DNA in either pre- or postflight urine samples, and that anti-CMV IgG antibody titers increased significantly for all shedders from each time point compared to their baseline values (Mehta et al., 2000b). In long duration spaceflight, $61 \%$ of astronauts shed CMV 
DNA in their urine during and after spaceflight in stark contrast to the absence of CMV DNA in urine samples taken 180 days before flight. These findings demonstrate that CMV reactivation occurs in astronauts regardless of mission duration, and this may pose additional threats to the health of crewmembers during longer-duration missions (Mehta et al., 2014, 2017).

\section{Rapid Detection of Virus for Application in Patient Populations}

The most obvious signs of VZV reactivation are the vesicular rash and the pain associated with zoster, however even in the absence of rash, the virus is active and can spread to the retina causing blindness, to the spinal cord causing paralysis and incontinence, and to cerebral arteries resulting in stroke (Kleinschmidt-DeMasters and Gilden, 2001; Orme et al., 2007). Associating VZV with a disease asymptomatically can be challenging. For example, when stroke occurs in the elderly, especially many months following zoster, the association with VZV reactivation requires cerebral spinal fluid (CSF) analysis for VZV antibodies (Nagel et al., 2007). Likewise, detection of asymptomatic VZV reactivation, which often is only seen as an increase in antibody titer against VZV (but may also result in virus transmission), is difficult to detect. In such instances, virological verification of $V Z V$ disease has relied on the detection of VZV DNA or anti-VZV IgG antibodies in CSF or, less often, the presence of VZV DNA in blood mononuclear cells or anti-VZV IgM antibodies in serum.

However, VZV DNA has been detected in the saliva samples from patients with acute zoster (Mehta et al., 2008), zoster sine herpete (Hato et al., 2000), chickenpox (Mehta et al., 2013b) and post-herpetic neuralgia (PHN) (Nagel et al., 2011) even before the rash appears, which now makes diagnosis less invasive and less time consuming. In fact, a rapid and sensitive virus detection method has been developed and used to detect virus in saliva samples taken from asymptomatic patients with neurologic and other VZV related disease (Mehta et al., 2013b). Figure 4 illustrates VZV copy numbers in saliva from shingles patients before anti-viral treatment. For this method, the saliva is collected by passive drool or by way of a synthetic swab and then processed for DNA within an hour from sample collection. The results from a few studies using this technique have shown that VZV DNA is present in $100 \%$ of patients tested before antiviral treatment and is exclusively in the cell pelleted fraction of saliva. These studies further showed that VZV, isolated from zoster patient saliva, was primarily associated with the epithelial cell membrane but could also be inside the cell. Epithelial cells with VZV continued to be present in the saliva of a single zoster patient up to 10 months after recovery. These kinds of studies are ongoing and our spaceflight-developed technology for rapid viral detection continues to be used locally and around the world for patients with zoster (Mehta et al., 2013b), chicken pox (Mehta et al., 2008), PHN (Nagel et al., 2011), multiple sclerosis (Ricklin et al., 2013), and various other neurological disorders (Gilden et al., 2010; Pollak et al., 2015).

\section{CONCLUSION}

Reactivation of latent viruses is a powerful biomarker of immune status for astronauts deployed to space. There are multiple factors that influence reactivation including increases in glucocorticoid/catecholamine secretion, cytokine profile shifts, and decreased function in the major leukocyte and lymphocyte subsets designed to suppress and eliminate viruses/virally infected cells. Viral reactivation is evident through the shedding of viral DNA in the body fluids of astronauts, and the viral load only increases with more time in space. Additionally, more than one virus generally reactivates at a time, potentially compounding the physiological ramifications of uncontrolled viral reactivation to not only rashes, but also severe target organ failures, and permanent vision and hearing loss. The occupational hazards for astronauts are profound, but research into the causes and mechanics of viral reactivation not only benefit the astronaut but also the general patient population. As our understanding of viral reactivation widens, we are better able to develop and implement effective countermeasures for our astronaut professionals, as well as targeted treatment regimens for immunocompromised individuals suffering the consequences of viral reactivation. As a result, this research has tremendous clinical relevance.

Ultimately, the information gleaned from these space studies will shape the way we prepare for and design exploration-class missions, beyond the moon and mars, where reactivation of latent viruses could result in increased risk for wide-ranging adverse medical events. Partial-gravity environments, e.g., on Mars, might be sufficient to curtail serious viral reactivation, but this needs to be addressed in future research. In the interim, because astronaut saliva contains increasingly significant viral DNA, during and after spaceflight that can be infectious, we recommend prophylactics (vaccines), where available, to the astronauts before they go into space.

\section{AUTHOR CONTRIBUTIONS}

SM designed and executed the study, collected and processed the samples from astronauts, analyzed the data, and wrote the manuscript. DP designed the study and wrote the manuscript. $\mathrm{BC}$ executed the study and wrote the manuscript. BR wrote the manuscript. ML contributed to the measurement of salivary cortisol and DHEA processing and manuscript preparation.

\section{FUNDING}

This work was supported by NASA (Grant Nos. 111-30-10-03 and 111-30-10-06) to DP.

\section{ACKNOWLEDGMENTS}

We gratefully acknowledge the conscientious participation of the astronauts in the study. 


\section{REFERENCES}

Bigley, A. B., Agha, N., Baker, F. L., Rezvani, K., Crucian, B. E., and Simpson, R. J. (2017). "Dysregulated NK-cell function during long-duration spaceflight," in Proceedings of the 13th ISEI Symposium, Coimbra.

Bravender, T. (2010). Epstein-Barr virus, cytomegalovirus, and infectious mononucleosis. Adolesc. Med. State Art Rev. 21, 251-264.

Christeff, N., Gherbi, N., Mammes, O., Dalle, M. T., Gharakhanian, S., Lortholary, O., et al. (1997). Serum cortisol and DHEA concentrations during HIV Infection. Psychoneuroendocrinology 22(Suppl. 1), S11-S18. doi: 10.1016/ S0306-4530(97)00015-2

Cohrs, R. J., Mehta, S. K., Schmid, D. S., Gilden, D. H., and Pierson, D. L. (2008). Asymptomatic reactivation and shed of infectious varicella zoster virus in astronauts. J. Med. Virol. 80, 1116-1122. doi: 10.1002/jmv.21173

Collins-McMillen, D., Kim, J. H., Nogalski, M. T., Stevenson, E. V., Chan, G. C., Caskey, J., et al. (2015). HCMV promotes survival of infected monocytes via a distinct temporal regulation of cellular Bcl-2 family proteins. J. Virol. 90, 2356-2371. doi: 10.1128/JVI.01994-15

Crucian, B., Babiak-Vazquez, A., Johnston, S., Pierson, D. L., Ott, C. M., Sams, C., et al. (2016). Incidence of clinical symptoms during long-duration orbital spaceflight. Int. J. Gen. Med. 9, 383-391. doi: 10.2147/IJGM.S114188

Crucian, B., and Sams, C. (2009). Immune system dysregulation during spaceflight: clinical risk for exploration-class missions. J. Leukoc. Biol. 86, 1017-1018. doi: 10.1189/jlb.0709500

Crucian, B., Simpson, R. J., Mehta, S., Stowe, R., Chouker, A., Hwang, S. A., et al. (2014). Terrestrial stress analogs for spaceflight associated immune system dysregulation. Brain Behav. Immun. 39, 23-32. doi: 10.1016/j.bbi.2014.01.011

Crucian, B., Stowe, R., Mehta, S., Uchakin, P., Quiriarte, H., Pierson, D., et al. (2013). Immune system dysregulation occurs during short duration spaceflight on board the space shuttle. J. Clin. Immunol. 33, 456-465. doi: 10.1007/s10875012-9824-7

Crucian, B., Stowe, R. P., Mehta, S., Quiriarte, H., Pierson, D., and Sams, C. (2015). Alterations in adaptive immunity persist during long-duration spaceflight. NPJ Microgravity 1:15013. doi: 10.1038/npjmgrav.2015.13

Crucian, B. E., Johnston, S., Mehta, S. K., Stowe, R., Uchakin, P., Quiriarte, H., et al. (2016). A case of persistent skin rash and rhinitis with immune system dysregulation onboard the international space station. J. Allergy Clin. Immunol. Pract. 4, 759-762.e8. doi: 10.1016/j.jaip.2015.12.021

Crucian, B. E., Zwart, S. R., Mehta, S. K., Uchakin, P., Quiriarte, H. D., Pierson, D. L., et al. (2014). Plasma cytokine concentrations indicate that in vivo hormonal regulation of immunity is altered during long-duration spaceflight. J. Interferon Cytokine Res. 34, 778-786. doi: 10.1089/jir.2013.0129

Eshleman, E., Shahzad, A., and Cohrs, R. J. (2011). Varicella zoster virus latency. Future Virol. 6, 341-355. doi: 10.2217/fvl.10.90

Furman, D., Jojic, V., Sharma, S., Shen-Orr, S. S., Angel, C. J. L., OnengutGumuscu, S., et al. (2015). Cytomegalovirus infection enhances the immune response to influenza. Sci. Transl. Med. 7:281ra43. doi: 10.1126/scitranslmed. aaa2293

Gilden, D., Cohrs, R. J., Mahalingam, R., and Nagel, M. A. (2010). Neurological disease produced by varicella zoster virus reactivation without rash. Curr. Top. Microbiol. Immunol. 342, 243-253. doi: 10.1007/82-2009-3

Glaser, R., Pearson, G. R., Bonneau, R. H., Esterling, B. A., Atkinson, C., and Kiecolt-Glaser, J. K. (1993). Stress and the memory T-cell response to the Epstein-Barr virus in healthy medical students. Health Psychol. 12, 435-442. doi: 10.1037/0278-6133.12.6.435

Goldstein, D. S. (2010). Adrenal responses to stress. Cell. Mol. Neurobiol. 30, 1433-1440. doi: 10.1007/s10571-010-9606-9

Grinde, B. (2013). Herpesviruses: latency and reactivation - viral strategies and host response. J. Oral Microbiol. 5, 1-9. doi: 10.3402/jom.v5i0.22766

Hato, N., Kisaki, H., Honda, N., Gyo, K., Murakami, S., and Yanagihara, N. (2000). Ramsay hunt syndrome in children. Ann. Neurol. 48, 254-256. doi: 10.1002/ 1531-8249(200008)48:2<254::AID-ANA17>3.0.CO;2-V

Kaur, I., Simons, E. R., Castro, V. A., Ott, C. M., and Pierson, D. L. (2005). Changes in monocyte functions of astronauts. Brain Behav. Immun. 19, 547-554. doi: $10.1016 /$ j.bbi.2004.12.006

Kempkes, B., and Robertson, E. S. (2015). Epstein-Barr virus latency: current and future perspectives. Curr. Opin. Virol. 14, 138-144. doi: 10.1016/j.coviro.2015. 09.007
Kleinschmidt-DeMasters, B. K., and Gilden, D. H. (2001). Varicella-zoster virus infections of the nervous system: clinical and pathologic correlates. Arch. Pathol. Lab. Med. 125, 770-780.

Mehta, S. K., Cohrs, R. J., Forghani, B., Zerbe, G., Gilden, D. H., and Pierson, D. L. (2004). Stress-induced subclinical reactivation of varicella zoster virus in astronauts. J. Med. Virol. 72, 174-179. doi: 10.1002/jmv.10555

Mehta, S. K., Crucian, B. E., Stowe, R. P., Simpson, R. J., Ott, C. M., Sams, C. F., et al. (2013a). Reactivation of latent viruses is associated with increased plasma cytokines in astronauts. Cytokine 61, 205-209. doi: 10.1016/j.cyto.2012.09.019

Mehta, S. K., Laudenslager, M. L., Stowe, R. P., Crucian, B. E., Sams, C. F., and Pierson, D. L. (2014). Multiple latent viruses reactivate in astronauts during space shuttle missions. Brain Behav. Immun. 41, 210-217. doi: 10.1016/j.bbi. 2014.05.014

Mehta, S. K., Tyring, S. K., Cohrs, R. J., Gilden, D. H., Feiveson, A. H., Lechler, K. J., et al. (2013b). Rapid and sensitive detection of varicella zoster virus in saliva of patients with herpes zoster. J. Virol. Methods 193, 128-130. doi: 10.1016/j.jviromet.2013.05.019

Mehta, S. K., Laudenslager, M. L., Stowe, R. P., Crucian, B. E., Feiveson, A. H., Sams, C. F., et al. (2017). Latent virus reactivation in astronauts on the international space station. NPJ Microgravity 3:11. doi: 10.1038/s41526-017-0015-y

Mehta, S. K., and Pierson, D. L. (2007). Reactivation of latent herpes viruses in cosmonauts during a Soyuz taxi mission. Microgravity Sci. Technol. 19, 215-218. doi: 10.1007/BF02919485

Mehta, S. K., Pierson, D. L., Cooley, H., Dubow, R., and Lugg, D. (2000a). EpsteinBarr virus reactivation associated with diminished cell-mediated immunity in antarctic expeditioners. J. Med. Virol. 61, 235-240.

Mehta, S. K., Stowe, R. P., Feiveson, A. H., Tyring, S. K., and Pierson, D. L. (2000b). Reactivation and shedding of cytomegalovirus in astronauts during spaceflight. J. Infect. Dis. 182, 1761-1764. doi: 10.1086/317624

Mehta, S. K., Tyring, S. K., Gilden, D. H., Cohrs, R. J., Leal, M. J., Castro, V. A., et al. (2008). Varicella-zoster virus in the saliva of patients with herpes zoster. J. Infect. Dis. 197, 654-657. doi: 10.1086/527420

Nagel, M. A., Choe, A., Cohrs, R. J., Traktinskiy, I., Sorensen, K., Mehta, S. K., et al. (2011). Persistence of varicella zoster virus DNA in saliva after herpes zoster. J. Infect. Dis. 204, 820-824. doi: 10.1093/infdis/jir425

Nagel, M. A., Forghani, B., Mahalingam, R., Wellish, M. C., Cohrs, R. J., Russman, A. N., et al. (2007). The value of detecting anti-VZV IgG antibody in CSF to diagnose VZV vasculopathy. Neurology 68, 1069-1073. doi: 10.1212/01.wnl. 0000258549.13334 .16

Nicoll, M. P., Proença, J. T., and Efstathiou, S. (2012). The molecular basis of herpes simplex virus latency. FEMS Microbiol. Rev. 36, 684-705. doi: 10.1111/j.15746976.2011.00320.x

Niedobitek, G., Meru, N., and Delecluse, H. J. (2001). Epstein-Barr virus infection and human malignancies. Int. J. Exp. Pathol. 82, 149-170. doi: 10.1046/j.13652613.2001.00190.x

O'Connor, C. M., and Murphy, E. A. (2012). A myeloid progenitor cell line capable of supporting human cytomegalovirus latency and reactivation, resulting in infectious progeny. J. Virol. 86, 9854-9865. doi: 10.1128/JVI.01278-12

Orme, H. T., Smith, A. G., Nagel, M. A., Bert, R. J., Mickelson, T. S., and Gilden, D. H. (2007). VZV spinal cord infarction identified by diffusion-weighted MRI (DWI). Neurology 69, 398-400. doi: 10.1212/01.wnl.0000266390.27177.7b

Padgett, D. A., and Glaser, R. (2003). How stress influences the immune response. Trends Immunol. 24, 444-448. doi: 10.1016/S1471-4906(03)00173-X

Pawelec, G. (2014). Immunosenenescence: role of cytomegalovirus. Exp. Gerontol. 54, 1-5. doi: 10.1016/j.exger.2013.11.010

Pierson, D. L., Mehta, S. K., and Stowe, R. P. (2007). Reactivation of latent herpes viruses in astronauts. Psychoneuroimmunology II, 851-868 doi: 10.1016/B978012088576-3/50047-2

Pierson, D. L., Stowe, R. P., Phillips, T. M., Lugg, D. J., and Mehta, S. K. (2005). Epstein-Barr virus shedding by astronauts during space flight. Brain Behav. Immun. 19, 235-242. doi: 10.1016/j.bbi.2004.08.001

Pollak, L., Mehta, S. K., Pierson, D. L., Sacagiu, T., Kalmanovich, S. A., and Cohrs, R. J. (2015). Varicella-zoster DNA in saliva of patients with meningoencephalitis: a preliminary study. Acta Neurol. Scand. 131, 417-421. doi: 10.1111/ane.12335

Reichelt, M., Zerboni, L., and Arvin, A. M. (2008). Mechanisms of varicella-zoster virus neuropathogenesis in human dorsal root ganglia. J. Virol. 82, 3971-3983. doi: 10.1128/JVI.02592-07 
Ricklin, M. E., Lorscheider, J., Waschbisch, A., Paroz, C., Mehta, S. K., Pierson, D. L., et al. (2013). T-cell response against varicella-zoster virus in fingolimod-treated MS patients. Neurology 81, 174-181. doi: 10.1212/WNL. 0b013e31829a3311

Sansoni, P., Vescovini, R., Fagnoni, F. F., Akbar, A., Arens, R., Chiu, Y. L., et al. (2014). New advances in CMV and immunosenescence. Exp. Gerontol. 55, 54-62. doi: 10.1016/j.exger.2014.03.020

Seitz, R. (2010). Human cytomegalovirus (HCMV)-revised. Transfus. Med. Hemother. 37, 365-375. doi: 10.1159/000322141

Stevenson, E. V., Collins-McMillen, D., Kim, J. H., Cieply, S. J., Bentz, G. L., and Yurochko, A. D. (2014). HCMV reprogramming of infected monocyte survival and differentiation: a goldilocks phenomenon. Viruses 6, 782-807. doi: 10.3390/v6020782

Stowe, R. P., Kozlova, E. V., Sams, C. F., Pierson, D. L., and Walling, D. M. (2011). Latent and lytic Epstein-Barr virus gene expression in the peripheral blood of astronauts. J. Med. Virol. 83, 1071-1077. doi: 10.1002/jmv.22079

Stowe, R. P., Pierson, D. L., and Barrett, A. D. T. (2001a). Elevated stress hormone levels relate to Epstein-Barr virus reactivation in astronauts. Psychosom. Med. 63, 891-895. doi: 10.1097/00006842-200111000-00007

Stowe, R. P., Pierson, D. L., and Barrett, A. D. T. (2001b). "Space flight-induced reactivation of latent Epstein-Barr virus," in Proceedings of the Conference and Exhibit on International Space Station Utilization, Cape Canaveral, FL.

Stowe, R. P., Sams, C. F., Mehta, S. K., Kaur, I., Jones, M. L., Feeback, D. L., et al. (1999). Leukocyte subsets and Neutrophil function after short-term spaceflight. J. Leukoc. Biol. 65, 179-186. doi: 10.1002/jlb.65.2.179
Thompson, M. P., and Kurzrock, R. (2004). Epstein-Barr virus and cancer. Clin. Cancer Res. doi: 10.1158/1078-0432.CCR-0670-3 [Epub ahead of print].

Varani, S., and Landini, M. (2011). Cytomegalovirus-induced immunopathology and its clinical consequences. Herpesviridae 2:6. doi: 10.1186/2042-4280-2-6

Webster, J. I., and Glaser, R. (2008). Stress hormones and immune Function. Cell. Immunol. 252, 16-26. doi: 10.1016/j.cellimm.2007.09.006

Wills, M. R., Poole, E., Lau, B., Krishna, B., and Sinclair, J. H. (2015). The immunology of human cytomegalovirus latency: could latent infection be cleared by novel immunotherapeutic strategies? Cell. Mol. Immunol. 12, 128138. doi: $10.1038 / \mathrm{cmi} .2014 .75$

Conflict of Interest Statement: BR was employed by GeoControl Systems Incorporated and SM was employed by Jes Tech, KBR Wyle Laboratories.

The remaining authors declare that the research was conducted in the absence of any commercial or financial relationships that could be construed as a potential conflict of interest.

Copyright (C) 2019 Rooney, Crucian, Pierson, Laudenslager and Mehta. This is an open-access article distributed under the terms of the Creative Commons Attribution License (CC BY). The use, distribution or reproduction in other forums is permitted, provided the original author(s) and the copyright owner(s) are credited and that the original publication in this journal is cited, in accordance with accepted academic practice. No use, distribution or reproduction is permitted which does not comply with these terms. 